\title{
Rituximab therapy for hairy cell leukemia: a retrospective study of 41 cases
}

\author{
Mathieu Leclerc • Felipe Suarez • Marie-Pierre Noël • Anne Vekhoff • Xavier Troussard • \\ Jean-François Claisse • Catherine Thieblemont • Frédéric Maloisel • Yves Beguin • \\ Jérôme Tamburini • Coralie Barbe • Alain Delmer
}

Received: 26 June 2014 / Accepted: 24 July 2014 / Published online: 7 September 2014

(C) Springer-Verlag Berlin Heidelberg 2014

\begin{abstract}
The purine analogs (PAs) cladribine and pentostatin have transformed the prognosis of hairy cell leukemia (HCL). However, some patients still relapse after PAs, or fail to reach an optimal response, and new agents are needed to further improve treatment outcome. We retrospectively studied 41 HCL patients from 10 centers in France and Belgium, who received 49 treatment courses with the anti-CD20 monoclonal antibody rituximab. Most of the patients were treated at relapse ( $84 \%$ of cases) and rituximab was combined to a PA in $41 \%$ of cases. Overall, response rate is $90 \%$ including $71 \%$ complete hematologic responses (CHRs). Frontline treatment, combination therapy, and absolute neutrophil count were
\end{abstract}

M. Leclerc $(\bowtie) \cdot$ A. Delmer

Centre Hospitalier Universitaire (CHU) de Reims, Hôpital Robert

Debré, Service d'Hématologie Clinique, Reims, France

e-mail: matleclerc@hotmail.fr

A. Delmer

e-mail: adelmer@chu-reims.fr

F. Suarez

Assistance Publique-Hôpitaux de Paris (AP-HP), Hôpital

Necker-Enfants Malades, Service d'Hématologie Adultes, Paris,

France

e-mail: felipe.suarez@nck.aphp.fr

\section{M.-P. Noël}

Centre Hospitalier Régional Universitaire de Lille,

Hôpital Claude Huriez, Service des Maladies du Sang,

Lille, France

e-mail: marie-pierre.noel@chru-lille.fr

A. Vekhoff

AP-HP, Hôpital Saint-Antoine, Service d'Hématologie,

Paris, France

e-mail: anne.vekhoff@sat.aphp.fr

X. Troussard

CHU de Caen, Hôpital Femme-Enfant-Hématologie, Service

d'Hématologie Clinique, Caen, France

e-mail: troussard-x@chu-caen.fr associated with response in multivariate analysis. Three-year relapse-free and overall survivals are 68 and $90 \%$, respectively. When combined to a PA, rituximab yields a $100 \%$ response rate, even beyond frontline therapy. In contrast, response rate is only $82 \%(59 \% \mathrm{CHR})$ when rituximab is used alone. In this latter setting, relapse rate is $56 \%$ and median time to relapse is 17.5 months. All eight patients who were treated two times with the antibody responded again to retreatment. We confirm the high efficacy of the combination rituximab + PA. However, when rituximab is used as monotherapy, response rate is lower and the high relapse rate is a concern. Prospective clinical trials are needed to confirm the

\section{J.-F. Claisse}

CHU d'Amiens, Hôpital Sud, Service d'Hématologie Clinique et de Thérapie Cellulaire, Amiens, France

e-mail: claisse.jean-francois@chu-amiens.fr

C. Thieblemont

AP-HP, Hôpital Saint-Louis, Service d'Hématologie-Oncologie,

Paris, France

e-mail: catherine.thieblemont@sls.aphp.fr

F. Maloisel

Groupe Hospitalier Saint-Vincent, Clinique Sainte-Anne, Service d'Oncologie et d'Hématologie, Strasbourg, France

e-mail: f.maloisel@solcrr.org

Y. Beguin

CHU of Liège and University of Liège, Liège, Belgium

e-mail: Yves.Beguin@chu.ulg.ac.be

J. Tamburini

AP-HP, Hôpital Cochin, Service d'Hématologie Clinique, Paris,

France

e-mail: jerome.tamburini@cch.aphp.fr

C. Barbe

CHU de Reims, Hôpital Robert Debré, Service de Coordination à la

Recherche Clinique, Reims, France

e-mail: cbarbe@chu-reims.fr 
superiority of the combination rituximab + PA over PA alone, both as frontline therapy and at relapse.

Keywords Hairy cell leukemia · Rituximab · Immunotherapy $\cdot$ Chronic leukemias

\section{Introduction}

Hairy cell leukemia (HCL) is a rare and indolent lymphoid disorder, representing $2 \%$ of all cases of lymphoid leukemias [1]. It is characterized by the presence of mature leukemic CD20-positive B cells showing both typical morphological features and phenotypic profile and accumulating in the bone marrow (BM), spleen, and liver. Hence, patients with HCL usually present with cytopenias, splenomegaly, and sometimes hepatomegaly [2]. Treatment of HCL relies mainly on the purine analogs (PAs) cladribine and pentostatin, which have shown similar efficacy and constitute the gold standard of care either as frontline therapy or for relapsed patients [3-7]. However, despite the remarkable response rates obtained with PA therapy, some patients will eventually relapse and the efficacy of these agents seems to decrease at each line of treatment [5]. The addition of new molecules to PAs may improve the response rates and prevent relapse.

Rituximab is a chimeric IgG1 kappa-type monoclonal antibody directed against the CD20 molecule. It was first used in relapsed patients with HCL more than 10 years ago [8], and several series of patients treated with rituximab as monotherapy were published in the following decade, reporting response rates ranging from 25 to $80 \%$ [9-13]. These encouraging results have led to combine rituximab with PA, both in previously untreated and in relapsed patients. As frontline therapy, combination with cladribine provided an impressive $100 \%$ response rate in two separate cohorts that included a total of 63 patients $[14,15]$. Moreover, molecular responses were obtained in $70 \%$ of these patients. In the relapse setting, the efficacy of rituximab combined with either cladribine, pentostatin, or even fludarabine is also substantial with similar response and relapse rates, even though the number of reported patients is much lower [16, 17].

We designed a multicenter retrospective study of patients treated with rituximab for HCL, either frontline or at relapse and either in monotherapy or in combination with other molecules. The objectives of the study were to assess the overall efficacy of rituximab in distinct clinical settings from a cohort of unselected patients representative of the population seen in the routine practice, and to identify prognostic factors for response, duration of response, and survival.

\section{Methods}

Patients

Patients were retrospectively recruited in 10 centers in France and Belgium from July 2002 to September 2012. They were eligible for inclusion in the study if they had a confirmed diagnosis of classical HCL and had received at least three subsequent injections of rituximab. No cases of HCL-variant were included. Diagnosis of HCL relied on identification of typical HCL cells in peripheral blood (PB) or BM with compatible immunophenotyping (diagnostic score of $3 / 4$ or $4 / 4$ according to Matutes et al. [18]) and/or immunochemistry.

\section{Rituximab therapy}

Rituximab was administered intravenously at the dose of $375 \mathrm{mg} / \mathrm{m}^{2}$ per injection in all patients but one who received two infusions of $1,000 \mathrm{mg}$ at day 1 and day 15 as induction therapy and then a consolidation phase with the classic dosage. Patients were allowed to receive several treatment sequences with rituximab during the course of their disease.

\section{Response assessment}

Response to rituximab therapy was assessed during the first 6 months following the end of treatment, depending on available data. Complete hematologic response (CHR) was defined as (i) the recovery of normal blood counts (absolute neutrophil count $(\mathrm{ANC}) \geq 1.5 \times 10^{9} / \mathrm{L}$, hemoglobin level $\geq 120 \mathrm{~g} / \mathrm{L}$ for men and $\geq 110 \mathrm{~g} / \mathrm{L}$ for women, and platelet count $\geq 100 \times 10^{9} /$ L) and (ii) the absence of circulating HCL cells and clinical signs (mainly splenomegaly and hepatomegaly) of HCL; depending on the availability of a BM assessment after rituximab therapy, CHR was further divided into three subgroups: stringent complete response (sCR) if BM evaluation showed no persistent leukemic cells, unconfirmed complete response ( $\mathrm{uCR}$ ) if no BM trephine biopsy or aspirate was performed after treatment, and CHR with persistent medullar infiltration by leukemic cells (iCHR). Partial response (PR) was defined as a $\geq 50 \%$ improvement for every CHR-defining criterion or normalization of at least one blood count (ANC, hemoglobin level, or platelet count), without circulating HCL cells. Patients not meeting criteria for at least PR were classified as non-responders (NR).

\section{Relapse}

Relapse was defined either by reappearance of HCL cells in $\mathrm{PB}$ or $\mathrm{BM}$ or by a significant cytopenia $\left(\mathrm{ANC} \leq 1.5 \times 10^{9} / \mathrm{L}\right.$, hemoglobin level $\leq 110 \mathrm{~g} / \mathrm{L}$, or platelet count $\leq 100 \times 10^{9} / \mathrm{L}$ ) arising during follow-up, without other identifiable cause. 
Time to relapse was defined as the time between the first rituximab infusion and relapse.

Minimal residual disease evaluation

Some patients included in this study had minimal residual disease (MRD) evaluation after treatment. For these patients, MRD was analyzed on a BM or PB sample using either immunophenotyping by flow cytometry or detection of an immunoglobulin heavy chain gene rearrangement by polymerase chain reaction (PCR) [19, 20].

Statistical analysis

Factors associated with treatment response were studied by univariate analysis (the Student, Wilcoxon, Chi-square, or Fisher's exact tests, as appropriate) and by multivariate analysis (binary logistic regression with ascending stepwise selection and entry and exit thresholds set at 0.10 ). All variables with a $p$ value $<0.10$ by univariate analysis were included in the multivariate analysis.

To assess potential predictive factors of relapse, univariate (log-rank tests) and multivariate (Cox stepwise regression with entry and removal limits set at 0.10 ) analyses were performed. All variables with a $p$ value $<0.10$ by univariate analysis were included in the multivariate analysis.

Overall survival (OS) and relapse-free survival (RFS) curves were estimated with the use of the Kaplan-Meier method. For patients who received two separate treatment sequences with rituximab, only the first one was included in the statistical analyses of prognostic factors and survival.

A $p$ value of 0.05 was considered statistically significant. All analyses were performed with $\mathrm{SAS}^{\circledR}$ software, version 9.3 (SAS Inc, Cary, NC, USA).

\section{Results}

Patients' characteristics

Forty-one patients were included in the study, for a total of 49 sequences of treatment with rituximab since eight of our patients were treated two times with this agent.

Patients' characteristics at time of rituximab therapy are summarized in Table 1. Most of them (76\%) were males, median age was 57 years, and one fifth was 70 or older. The median time elapsed between diagnosis and initiation of rituximab therapy was more than 6 years (range 0-32 years).

Rituximab was used as part of frontline therapy in $16 \%$ of cases ( 8 of 49 treatments performed). For the 41 remaining cases, the median number of previous therapeutic lines was 3 (range 1-8) with cladribine and pentostatin being the most
Table 1 Patients' characteristics $(n=41)$

\begin{tabular}{ll}
\hline Sex ratio M/F & $31 / 10$ \\
\hline Age at diagnosis (median, range) & 49 years $(27-84)$ \\
Age at first rituximab infusion (median, range) & 57 years $(28-91)$ \\
Performance status $0 / 1$ & $75.6 \% / 19.5 \%$ \\
$2 />2$ & $4.9 \% / 0 \%$ \\
B symptoms & $8(17 \%)$ \\
Splenomegaly & $4(8.2 \%)$ \\
Leukocytes $\left(\times 10^{9} / \mathrm{L}\right)$ (median, range) & $2.1(0.5-16.8)$ \\
Neutrophils $\left(\times 10^{9} / \mathrm{L}\right)$ (median, range) & $1(0.1-5.4)$ \\
Monocytes $\left(\times 10^{9} / \mathrm{L}\right)($ median, range) & $0.04(0-0.6)$ \\
Hemoglobin $(\mathrm{g} / \mathrm{L})($ median, range) & $117.5(50-169)$ \\
Platelets $\left(\times 10^{9} / \mathrm{L}\right)($ median, range $)$ & $94(21-386)$ \\
Presence of circulating HCL cells $(\%$ of cases) & $39.1 \%$ \\
\hline
\end{tabular}

$M$ males, $F$ females, $H C L$ hairy cell leukemia

frequent previously used drugs (78 and $54 \%$ of cases, respectively). All these patients had received at least one PA, and in $32 \%$ of cases, they had received both cladribine and pentostatin. Other previous therapies included interferon in 20 cases $(49 \%)$, splenectomy in 7 cases $(17 \%)$, norethandrolone in 1 case $(2 \%)$, and, as mentioned above, rituximab in 8 cases $(20 \%)$.

For relapsed patients, overall response rate (ORR) to the therapeutic line immediately preceding rituximab (which included a PA in $76 \%$ of cases) was $87 \%$ (60\% CHR and $27 \%$ PR).

\section{Rituximab therapy}

The median number of rituximab infusions per treatment course was 4 (range 3-12). Most of the patients received either four or eight infusions (65 and $15 \%$ of cases, respectively). Rituximab was used as monotherapy in $55 \%$ of cases and in combination with other agents in $45 \%$ of cases. The most frequently associated molecule was cladribine (18 of 22 cases, $82 \%$ ), which was given either before ( 7 cases), concomitantly ( 8 cases), or after rituximab administration (3 cases). In the remaining cases, rituximab was combined with pentostatin (two patients), interferon (one patient), and steroids (one patient). Rituximab was administered weekly in $88 \%$ of cases (among whom $23 \%$ subsequently received additional infusions at longer intervals). Median rituximab treatment duration was 24 days (range 14-695).

Rituximab was given because of disease relapse or progression (66\% of cases), contraindication to PA (12\%), failure of previous therapeutic line $(10 \%)$, detectable MRD after PA (10 \%), and associated auto-immune hemolytic ane$\operatorname{mia}(2 \%)$. 


\section{Efficacy}

Response assessment was available in 48 cases (Table 2). Whatever treatment modalities (monotherapy or combination), ORR was $90 \%$ (43 of 48 cases) and CHR was achieved in 34 cases (71\%), including 6 sCRs (13\%), 26 uCRs (54\%), and 2 iCHRs (4\%). PR was observed in nine cases (19\%) and treatment failure in five cases $(10 \%)$. Bone marrow evaluation was done in only 14 cases ( $29 \%$ ), mostly by BM aspirate (only three patients had a trephine biopsy), and was normal in $43 \%$ of them.

All eight patients who received rituximab during frontline therapy achieved a CHR (three sCRs and five uCRs), even though it was combined with cladribine for five of them.

Regarding treatments given in relapsed patients, ORR was $88 \%$ (including $65 \% \mathrm{CHR}$ and $23 \% \mathrm{PR}$ ), with lower response rates when rituximab was used as monotherapy (24 cases, ORR $79 \%$ and CHR $54 \%$ ) than when it was combined with cladribine or pentostatin (15 cases, ORR $100 \%$, CHR $86 \%$ ). Of note, all five treatment failures observed in this study were relapsed patients treated with rituximab alone.

Overall, when rituximab was used in combination with a PA (20 cases), ORR was $100 \%$ (including $90 \% \mathrm{CHR}$ ) versus $82 \%$ (59\% CHR) when used as monotherapy (27 cases). No significant association was found between the number of administered rituximab infusions and the probability of response to treatment; particularly, ORR for patients treated with four infusions ( $90 \%$, including $71 \%$ CHR) was comparable to that of patients receiving more than four infusions (93\%, including $73 \% \mathrm{CHR}$ ).

Interestingly, all eight patients who had previously been treated with rituximab responded to re-treatment with this agent ( $6 \mathrm{uCRs}, 1 \mathrm{iCHR}$, and $1 \mathrm{PR})$. Among them, five patients even had a better response at re-treatment, although for only one of them, rituximab was combined with a PA for this second treatment course.

Five parameters correlated with the likelihood of achieving a response to rituximab were identified by univariate analysis: first-line treatment, combination therapy, ANC and platelet count (with higher numbers increasing the probability of achieving a response), and percentage of HCL cells in PB before treatment (with lower numbers increasing the probability of achieving a response). Three independent factors persisted after multivariate analysis: first-line treatment (odds ratio $(\mathrm{OR})=0.027,95 \%$ confidence interval $(\mathrm{CI})=[0.001-$ $0.555], p=0.0192)$, combination therapy ( $\mathrm{OR}=10.120,95 \%$ $\mathrm{CI}=[1.227-83.485], p=0.0316)$, and high ANC before treatment $(\mathrm{OR}=1.002,95 \% \mathrm{CI}=[1.001-1.004], p=0.0060)$.

Nine patients had MRD evaluation after rituximab therapy, which was performed by immunophenotyping in six cases and by PCR in three cases. Two of them had undetectable disease. Significant diminution of MRD was observed in three additional cases, whereas three patients had stable MRD. The last patient had a very low positive MRD after rituximab, close to the detection threshold, but pre-treatment MRD was not available.

\section{Relapses and duration of response}

Among the 43 responses observed after rituximab therapy either alone or in combination, relapse or progression occurred in 15 cases ( $35 \%$ ), after a median duration of 19 months (range 2-39) since the first rituximab infusion (Table 2). Three-year RFS was 68 \% (Fig. 1).

Regarding the eight patients who received rituximab during frontline therapy (median follow-up 30.5 months, range 476), only $1(13 \%)$ relapsed at 38 months. On the other hand, when rituximab was used beyond frontline therapy (median follow-up 38 months, range 5-117), relapse rate was $40 \%$ and median time to relapse was only 17.5 months (range 2-39). Concerning the eight patients who were re-treated with rituximab, five relapsed after this second treatment (63\%), at a median of 30 months.

When rituximab was given alone, the relapse rate was as high as $56 \%$ compared to $14 \%$ when used in combination with another drug (only $11 \%$ when the other drug was a PA). Time to relapse was also shorter in case of monotherapy (median 17.5 vs 38 months).

The only independent prognostic factors for relapse/ progression identified by multivariate analysis were platelet
Table 2 Response to treatment, relapse rate, and duration of response

$R$ rituximab, $P A$ purine analog, $O R R$ overall response rate, $C H R$ complete hematologic response, mo months

\begin{tabular}{cllllc}
\hline & Number & ORR (\%) & CHR (\%) & $\begin{array}{l}\text { Relapse } \\
\text { rate (\%) }\end{array}$ & $\begin{array}{c}\text { Duration of response } \\
\text { (mo) (median, range) }\end{array}$ \\
\hline Overall & 48 & 90 & 71 & 35 & $26(2-113)$ \\
1 st line & 8 & 100 & 100 & 13 & $30.5(4-54)$ \\
R alone & 3 & 100 & 100 & 0 & $40(12-54)$ \\
R + PA & 5 & 100 & 100 & 20 & $29(4-38)$ \\
Relapse & 40 & 88 & 65 & 40 & $23(2-113)$ \\
R alone & 24 & 79 & 54 & 63 & $23(7-113)$ \\
R + PA & 14 & 100 & 86 & 7 & $24.5(10-79)$ \\
R + other & 2 & 100 & 50 & 50 & $21(2-40)$ \\
\hline
\end{tabular}


Fig. 1 Relapse-free survival. Pts patients, flup follow-up

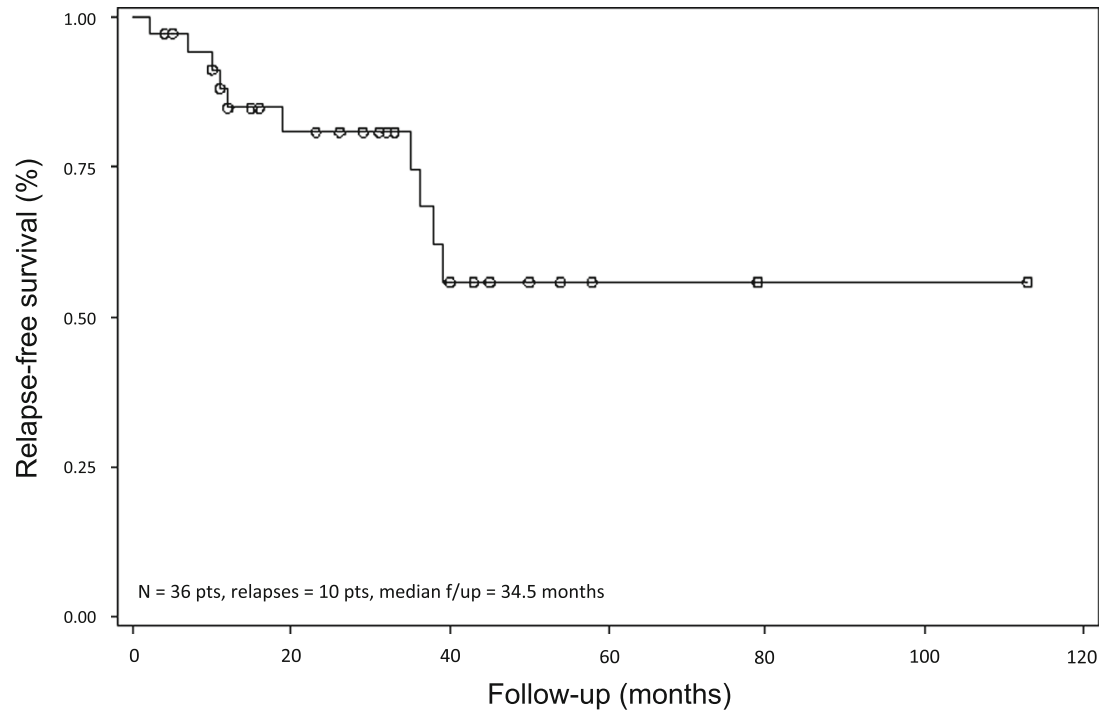

infusion requirement before rituximab therapy (hazard ratio $(\mathrm{HR})=17.783,95 \% \mathrm{CI}=[1.112-284.486], p=0.0419)$ and hemoglobin level after treatment (with lower numbers increasing the probability of relapse $)(\mathrm{HR}=0.943,95 \% \mathrm{CI}=[0.892-$ 0.998], $p=0.0406$ ).

\section{Overall survival}

After a median follow-up of 36 months (range 4-117), 6 of 41 patients had died (15\%). Death occurred after a median of 37 months (range 9-76) since first rituximab infusion. Threeyear OS for the whole cohort was $90 \%$ (Fig. 2). All of six patients either did not respond to treatment or relapsed, except one who died in remission.

\section{Discussion}

Whereas the largest series published to date concerns the use of rituximab in combination with PA for frontline therapy of HCL $[14,15]$, this situation represents only 5 of the 49 treatments reported in our study, with an excellent treatment outcome (100\% CHR). Most of our patients were treated at relapse, after multiple courses of therapy and a median time of more than 6 years since HCL diagnosis. This can explain the low incidence of splenomegaly, B symptoms, and severe cytopenias at time of initiation of rituximab treatment. In this relapse setting, the response rate we report ( $88 \%$ ORR including $65 \%$ CHR) is satisfactory and equivalent to the one observed at the previous line of therapy, even though combination to PA seems to provide a real benefit in this patient
Fig. 2 Overall survival. Pts patients, f/up follow-up

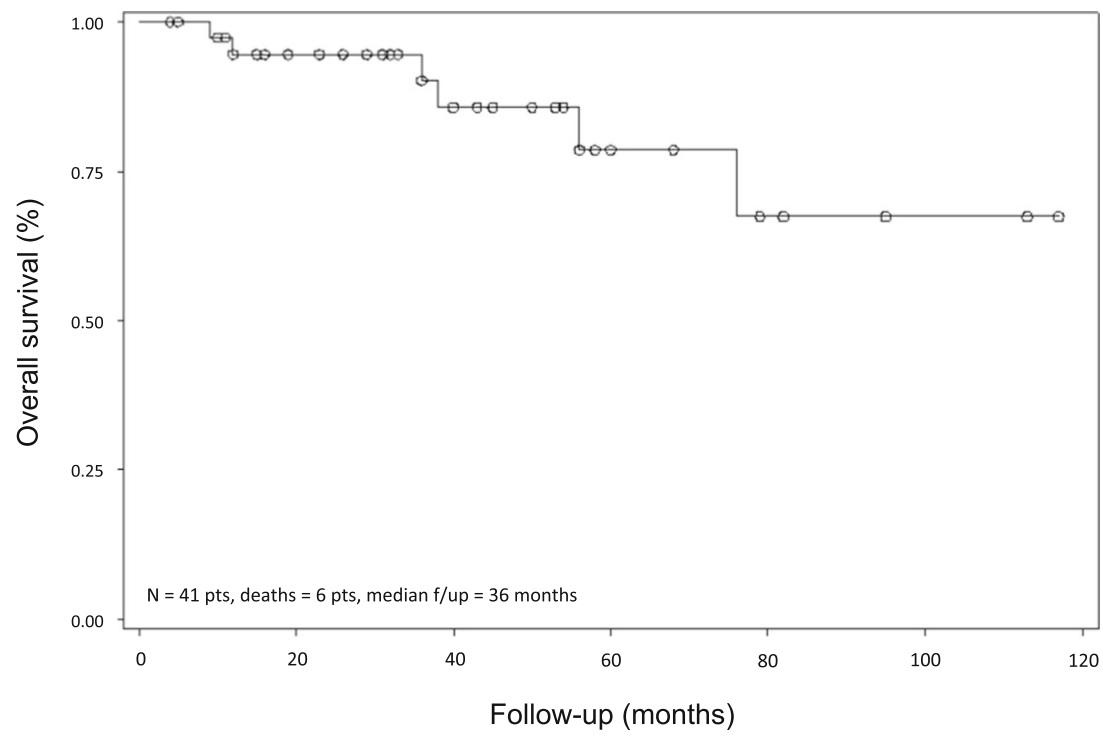


population. There is scarce data available concerning the use of frontline rituximab as monotherapy. Only four cases have been reported to date and two of these patients have responded to treatment $[10,21]$. Here, we report three additional cases of patients not eligible for frontline PA who received rituximab alone. They all met uCR criteria and none of them had relapsed after 12, 40, and 54 months of follow-up. Thus, this treatment approach is feasible and might represent a good alternative to interferon for patients who have contraindication to PA, even though more data are needed to confirm our results.

Another interesting issue that has not been assessed in the literature is whether or not patients who have already received rituximab for HCL may benefit from re-treatment with this agent. In fact, only one case has been reported so far [22]. Eight of the 41 patients of our study were treated two times with rituximab during the course of their disease. The response rate at re-treatment is excellent $(100 \%$ ORR including $88 \%$ CHR), and most surprisingly, five of these eight patients had a better response to re-treatment as compared with first treatment with rituximab, and none of them had a worst response to re-treatment. Although the number of cases is low, these results seem to support the possibility of multiple rituximab therapy during the course of HCL, even in case of non-optimal response to first treatment.

Despite the good response rate reported in our study, relapse, which occurred in one third of our patients, remains a concern, mostly when rituximab is used alone and beyond frontline therapy. In this latter situation, the relapse rate we observe $(63 \%)$ is higher than the ones previously reported in the literature (ranging from 0 to $50 \%$ ) [9-13]. The longest follow-up in our cohort (median 45 months for these patients) may explain this difference. However, even in case of combination with PA, relapse rate in our study (11\% after a median follow-up of 24.5 months) is still higher than published data, although to a lesser extent $[15,16]$.

This study is the largest cohort of patients treated with rituximab for HCL reported to date. The large inclusion criteria we used have allowed us to study this molecule in a wide range of clinical situations, some of which have barely been explored in the literature. Most of all, our patients are not selected patients for prospective clinical trials but likely represent a good sample of the population met in routine clinical practice. On the other hand, one may argue that a limitation of this study is the wide heterogeneity of our cohort. Additionally, our data do not allow us to draw firm conclusions regarding the role of rituximab for MRD eradication. In fact, only very few patients had an MRD evaluation after treatment. Moreover, results are difficult to interpret because of the heterogeneity of the techniques and samples used for each patient. The low rate of MRD negativity we observe after rituximab (two of nine patients) may also be due to the fact that MRD evaluation was often performed quite early after treatment, whereas previously published data have shown that molecular responses increase over time and are still quite low at 2 and even 6 months $[19,14]$.

One of the limitations of this work may also concern the choice of response criteria, which are slightly different from the ones published in the guidelines from the British Committee for Standards in Haematology [6]. In these guidelines, response assessment and distinction between PR and CR rely mainly on BM evaluation. However, according to the recently published recommendations of the French Society of Hematology for the management of HCL patients [23], BM trephine biopsy evaluation after treatment is not mandatory in France and is barely realized outside prospective clinical trials. Indeed, in our retrospective study, less than a third of patients had a $\mathrm{BM}$ evaluation after rituximab therapy (and only three had a BM trephine biopsy), making response assessment incongruous in $70 \%$ of cases regarding the British guidelines. Therefore, one should keep in mind that the response rate we report may have been overestimated by the criteria we used and the fact that most patients did not have $\mathrm{BM}$ evaluation. On the other hand, as it has already been shown that the optimal response may not occur earlier than 2 or 3 months after treatment, response rate may also have been underestimated in a few cases in which response assessment was done too early because of insufficient follow-up or missing data. We believe, however, that the criteria we chose are well adapted to routine clinical practice as they are based on the concept of CHR, which is easy to assess. Another limitation of this study is the relatively short median follow-up (36 months) compared to other published series of patients suffering from this chronic disease.

In conclusion, regarding the results of this and previous studies, and before further data is available concerning the efficacy and toxicity of BRAF inhibitors, we think rituximab therapy for HCL should be considered either frontline, i.e., for patients not eligible for treatment with a PA or not achieving CR or negative MRD after PA, or at relapse, in combination with a PA whenever possible given the high relapse rate and short response duration when used as monotherapy. Randomized prospective studies are warranted to confirm the superiority of the combination rituximab + PA over PA alone.

Conflict of interest The authors declare that they have no conflict of interest.

Authors' contribution $\mathrm{ML}$ and $\mathrm{AD}$ designed the study; ML, FS, MPN, AV, XT, JFC, CT, FM, YB, JT, and AD provided the data; ML and $\mathrm{CB}$ performed the statistical analysis; ML and AD wrote the paper; all authors read and approved the final manuscript and agreed to submission. 


\section{References}

1. Foucar K, Falini B, Catovsky D, Stein H (2008) Hairy cell leukaemia, vol 1. WHO classification of tumours of haematopoietic and lymphoid tissues. IARC Press, Lyon

2. Bouroncle BA (1979) Leukemic reticuloendotheliosis (hairy cell leukemia). Blood 53(3):412-436

3. Spiers AS, Moore D, Cassileth PA, Harrington DP, Cummings FJ, Neiman RS, Bennett JM, O'Connell MJ (1987) Remissions in hairycell leukemia with pentostatin (2'-deoxycoformycin). N Engl J Med 316(14):825-830

4. Piro LD, Carrera CJ, Carson DA, Beutler E (1990) Lasting remissions in hairy-cell leukemia induced by a single infusion of 2-chlorodeoxyadenosine. N Engl J Med 322(16):1117-1121

5. Else M, Dearden CE, Matutes E, Garcia-Talavera J, Rohatiner AZ, Johnson SA, O'Connor NT, Haynes A, Osuji N, Forconi F, Lauria F, Catovsky D (2009) Long-term follow-up of 233 patients with hairy cell leukaemia, treated initially with pentostatin or cladribine, at a median of 16 years from diagnosis. Br J Haematol 145(6):733-740

6. Jones G, Parry-Jones N, Wilkins B, Else M, Catovsky D (2012) Revised guidelines for the diagnosis and management of hairy cell leukaemia and hairy cell leukaemia variant*. Br J Haematol 156(2): 186-195

7. Flinn IW, Kopecky KJ, Foucar MK, Head D, Bennett JM, Hutchison R, Corbett W, Cassileth P, Habermann T, Golomb H, Rai K, Eisenhauer E, Appelbaum F, Cheson B, Grever MR (2000) Longterm follow-up of remission duration, mortality, and second malignancies in hairy cell leukemia patients treated with pentostatin. Blood 96(9):2981-2986

8. Hagberg H (1999) Chimeric monoclonal anti-CD20 antibody (rituximab) - an effective treatment for a patient with relapsing hairy cell leukaemia. Med Oncol 16(3):221-222

9. Lauria F, Lenoci M, Annino L, Raspadori D, Marotta G, Bocchia M, Forconi F, Gentili S, La Manda M, Marconcini S, Tozzi M, Baldini L, Zinzani PL, Foa R (2001) Efficacy of anti-CD20 monoclonal antibodies (Mabthera) in patients with progressed hairy cell leukemia. Haematologica 86(10):1046-1050

10. Hagberg H, Lundholm L (2001) Rituximab, a chimaeric anti-CD20 monoclonal antibody, in the treatment of hairy cell leukaemia. $\mathrm{Br} \mathrm{J}$ Haematol 115(3):609-611

11. Nieva J, Bethel K, Saven A (2003) Phase 2 study of rituximab in the treatment of cladribine-failed patients with hairy cell leukemia. Blood 102(3):810-813

12. Thomas DA, O'Brien S, Bueso-Ramos C, Faderl S, Keating MJ, Giles FJ, Cortes J, Kantarjian HM (2003) Rituximab in relapsed or refractory hairy cell leukemia. Blood 102(12):3906-3911
13. Zenhausern R, Simcock M, Gratwohl A, Hess U, Bargetzi M, Tobler A (2008) Rituximab in patients with hairy cell leukemia relapsing after treatment with 2-chlorodeoxyadenosine (SAKK 31/98). Haematologica 93(9): 1426-1428

14. Cervetti G, Galimberti S, Andreazzoli F, Fazzi R, Cecconi N, Caracciolo F, Petrini M (2008) Rituximab as treatment for minimal residual disease in hairy cell leukaemia: extended follow-up. Br J Haematol 143(2):296-298

15. Ravandi F, O'Brien S, Jorgensen J, Pierce S, Faderl S, Ferrajoli A, Koller C, Challagundla P, York S, Brandt M, Luthra R, Burger J, Thomas D, Keating M, Kantarjian H (2011) Phase 2 study of cladribine followed by rituximab in patients with hairy cell leukemia. Blood 118(14):3818-3823

16. Else M, Dearden CE, Matutes E, Forconi F, Lauria F, Ahmad H, Kelly S, Liyanage A, Ratnayake V, Shankari J, Whalley I, Catovsky D (2011) Rituximab with pentostatin or cladribine: an effective combination treatment for hairy cell leukemia after disease recurrence. Leuk Lymphoma 52(Suppl 2):75-78

17. Gerrie AS, Zypchen LN, Connors JM (2012) Fludarabine and rituximab for relapsed or refractory hairy cell leukemia. Blood 119(9): 1988-1991

18. Matutes E, Morilla R, Owusu-Ankomah K, Houliham A, Meeus P, Catovsky D (1994) The immunophenotype of hairy cell leukemia (HCL). Proposal for a scoring system to distinguish HCL from B-cell disorders with hairy or villous lymphocytes. Leuk Lymphoma 14(Suppl 1):57-61

19. Cervetti G, Galimberti S, Andreazzoli F, Fazzi R, Cecconi N, Caracciolo F, Petrini M (2004) Rituximab as treatment for minimal residual disease in hairy cell leukaemia. Eur J Haematol 73(6):412417

20. Matutes E, Meeus P, McLennan K, Catovsky D (1997) The significance of minimal residual disease in hairy cell leukaemia treated with deoxycoformycin: a long-term follow-up study. Br J Haematol 98(2): 375-383

21. Malfuson JV, Doghmi K, Fagot T, Souleau B, De Revel T, Nedellec G (2006) Efficacy of rituximab in hairy cell leukemia. Presse Med 35(5 Pt 1):801-802

22. Buckstein R, Patel H, Chesney A, Reis M, Imrie K (2006) Multiply relapsing hairy cell leukemia responsive to repeated courses of rituximab: a case report. Hematology 11(4):267270

23. Cornet E, Delmer A, Feugier P, Garnache-Ottou F, Ghez D, Leblond V, Levy V, Maloisel F, Re D, Zini J, Troussard X (2014) Recommendations of the SFH (French Society of Haematology) for the diagnosis, treatment and follow-up of hairy cell leukemia. Ann Hematol In press 